Pacific Journal of Mathematics

TOPOLOGICAL RINGS WITH PROPERTY (Y) 


\title{
TOPOLOGICAL RINGS WITH PROPERTY (Y)
}

\author{
W. E. BAXTER
}

This paper continues the study of Jordan ideals of the symmetric elements, $S$, of a 2-torsion free semiprime ring, $A$, with $2 A=A$. Previous results assuming proper involution, as well as the annihilator condition on certain right ideals in topological rings with property $(Y)$, are shown to be too restrictive. Finally, it is shown that every Jordan ideal of $S$ either contains an idempotent, or is contained in the annihilator of the socle.

We denote the involution by $x \rightarrow x^{*}$, and let $(a, b)_{J}=a b+b^{*} a^{*}$ and $(a, b)_{L}=a b-b^{*} a^{*}$ for all $a, b \in A$. In [1], we observed that $B=$ $\left\{b \mid(b, a)_{J} \in U\right.$, a Jordan ideal of $S$, for all $\left.a \in A\right\}$ is a right ideal, that $S B \subseteq B, U \circ U \subseteq B$, and $\mathscr{L}(B) S \subseteq \mathscr{L}(B)$. This is the notation of [1].

In [2], Herstein proves that if $A$ is semiprime then there exists no nonzero left or right nil ideals of bounded index of nilpotency. In [1], we proved that if $U$ is a Jordan ideal of $S$ and $u^{2}=\theta$ for all $u \in U$ then $U=(\theta)$. We now generalize this result to the analog of the Herstein result.

Theorem 1. Let $U$ be a Jordan ideal of $S$. Assume that there exists an integer $k$ such that $u^{k}=\theta$ for all $u \in U$, then $U=(\theta)$.

The theorem is proved in [1] for $k=2$. It is sufficient by induction argument to show that $u^{4 N}=\theta$ for all $u \in U$ implies $u^{2 N}=\theta$; for then, $u^{4}=\theta$ for all $u \in U$ implies $U=(\theta)$, while for $k \geqq 5$, then 4 is a factor of $k, k+1, k+2$, or $k+3$ and so $2 N<k$ and by induction we are done. Now, $u \in U$, implies $u^{2 N} \in B$ and so assuming $v^{4 N}=\theta$ for all $v \in U$, we have for each $a \in A, \theta=u^{2 N}\left(u^{2 N} a+a^{*} u^{2 N}\right)^{4 N}=$ $u^{2 N}\left(a^{*} u^{2 N}\right)^{4 N}$ and therefore, $\left(u^{2 N} a\right)^{4 N+1}=\theta$ or $u^{2 N}=\theta$.

We note next that if $b$ and $c$ are in $B$ and $r \in A$, then both $r c^{*} b=(c, r)_{J} b-c r^{*} b \in B$ and $c^{*} b=\left(c+c^{*}\right) b-c b \in B$. We make use of these facts in the following theorem which is the main result of $\S 2,[1]$.

Theorem 2. $\mathscr{L}(B)$ is a self-adjoint two-sided ideal in $A$.

We need only show that $\mathscr{L}(B)$ is a right ideal. Using the above remark $\left(a b^{*} b \in B\right.$ for all $\left.b \in B, a \in A\right)$ we see that as

$$
x\left((b, a)_{J}\right)^{2}=\theta
$$


for all $a \in A, b \in B, x \in \mathscr{L}(B)$, then

$$
x\left(a^{*} b^{*}\right)^{2}=\theta .
$$

For $x \in \mathscr{L}(B), b \in B, x b^{*}=x\left(b+b^{*}\right) \in \mathscr{L}(B)$. By (I) and the fact that $B^{*}$ is a left ideal, we have $\left(x b^{*}\right)\left[a^{*}\left(x b^{*}\right)\right]^{2}=\theta$ for all $a \in A$. Thus, $x b^{*} A$ is nil of bounded index 3 , and so $x b^{*}=\theta$. As $B^{*}$ is a left ideal, $x a b^{*}=\theta$ for all $a \in A$. This means $x^{*} b=\left(b^{*} x\right)^{*}=\theta^{*}=\theta$. Therefore, $\mathscr{L}(B)$ is self-adjoint and hence is a two-sided ideal.

Lemma 3. $\mathscr{L}(B) \cap B=(\theta)$.

$\mathscr{L}(B)$ being two-sided implies that $\mathscr{L}(B) \cap B$ is a nilpotent right ideal and so is $(\theta), A$ being a semiprime ring.

It now follows immediately that.

$$
\text { Lemma 4. } U \cap \mathscr{L}(B)=(\theta) \text {. }
$$

One observes that $b S b^{*}$ and $u S u$ are in $B \cap U$ for all $b \in B$ and $u \in U$. We make use of these facts to prove.

Theorem 5. $\mathscr{L}(B)=\mathscr{L}(B \cap S)=\mathscr{L}(B \cap U)=\mathscr{L}(U)$.

The proper order of set inclusions for the first three of these sets are obvious. Thus the theorem is proved if we show $\mathscr{L}(U) \subseteq \mathscr{L}(B)$ and $\mathscr{L}(B \cap U) \subseteq \mathscr{L}(U)$.

Let $x \in \mathscr{L}(U)$ and $b \in B$ then $x b x b x=x b x\left(b x+x^{*} b^{*}\right)-x\left(b x x^{*} b^{*}\right)=$ $\theta$. Therefore, $\mathscr{L}(U) b$ is a nil left ideal of index 3 , leading to the conclusion that $\mathscr{L}(U) \subseteq \mathscr{L}(B)$.

Similary, if $x \in \mathscr{L}(B \cap U)$ and $u \in U$ then

$$
x u x u x u=x\left[u\left(x+x^{*}\right) u\right] x u-x u\left(x^{*} u x\right) u=\theta,
$$

which shows that $\mathscr{L}(B \cap U) \subset \mathscr{L}(U)$.

We draw as an immediate consequence,

Corollary 6. $\mathscr{L}(S)=(\theta)$.

Now, define

Definition 7. $U_{J}=\left\{s \in S \mid(s, u)_{J}=\theta\right.$ for all $\left.u \in U\right\}$.

We wish to conclude that

Theorem 8. $U_{J}$ is a Jordan ideal of $S$ and $U_{J}=\mathscr{L}(U) \cap S$. 
Let $s \in U_{J}, u \in U$ then $(s, u)_{J}=\theta=\left(s, u^{2}\right)_{J}$ : The left equality yields $s u=-u s$ and so $2 s u^{2}=\theta$, or by hypothesis on $A, s u^{2}=\theta$. Thus,

$$
u s u=\theta .
$$

Now, let $s \in U_{J}, u \in B \cap U$, and $a \in A$. Noting that suasu= $s(u, a)_{J} s u$ we conclude that

$$
(s u a)^{3}=s\left[(u, a)_{J} s(u, a)_{J}\right] s u a=\theta .
$$

Thererfore, $s \in \mathscr{L}(B \cap U)=\mathscr{L}(U)$. Thus, $U_{J} \subseteq \mathscr{L}(U) \cap S$.

However, $\mathscr{L}(U) \cap S \subseteq U_{J}$ is immediate. As the equality holds we conclude that $U_{J}$ is a Jordan ideal.

Two immediate corollaries are important for later theorems.

Corollary 9. If $U_{J}=(\theta)$, then $\mathscr{f}(U)=(\theta)$.

As $\mathscr{L}(U)$ is self-adjoint, the hypothesis implies $\mathscr{L}(U) \subseteq K$ and so the desired conclusion.

Corollary 10. $T=\left\{t \in S \mid(t, s)_{J} \in U\right.$ for all $\left.s \in S\right\}$ is a Jordan ideal and $T_{J}=U_{J}=(B \cap S)_{J}$.

It follows immediately that $T$ is a Jordan ideal of $S$ and that $T_{J} \subseteq(B \cap S)_{J}$. By Theorem 8,

$$
U_{J}=\mathscr{L}(U) \cap S=\mathscr{P}(B \cap S) \cap S=(B \cap S)_{J} .
$$

Let $r \in(B \cap S)$, and $t \in T$ then

$$
(r, t)_{,} \in(B \cap S)_{J} \cap U \subseteq \mathscr{L}(U) \cap U=(\theta) .
$$

Thus, $r \in T_{J}$ and the corollary is proved.

2. Topological rings with property (Y). In [1] we defined these rings. Because of the results of the previous section, we now modify that definition by removing the condition of proper involution and by defining the annihilator condition for Jordan ideals based wholly on elements in $S$.

Definition 11. A semiprime topological ring $A$ with involution $\left(x \rightarrow x^{*}\right)$ is called a topological ring with property $(Y)$ if, and only if,

(1) $A=2 A$, any net $\left\{2 x_{\alpha}\right\} \rightarrow \theta$ implies $\left\{x_{\alpha}\right\} \rightarrow \theta$, and $A^{3}$ is dense in $A$ (the latter is true if the socle is dense in $A$ );

(2) the involution is continuous;

(3) $U_{J}=(\theta)$, for a closed Jordan ideal $U$ of $S$, if, and only if, $U=S$. 
A part of our previous definition had $\mathscr{L}(U)=(\theta)$ if, and only if, $U=S$ for closed Jordan ideals $U$ of $S$. We now see that Theorem 8 and Corollary 9 yield this equivalent formulation. By Corollary 6, $\mathscr{L}(S)=(\theta)$ in a semiprime ring. Hence, we see that the restriction (3) really asserts that for proper closed Jordan ideals $U, U_{J} \neq(\theta)$.

Although we have introduced the notion of a topological ring with property $(Y)$, our next theorem is stated for less restrictive $A$ since this form of the theorem is used in the next section.

THEOREM 12. Let $A$ be a semiprime topological ring with continuous involution, $2 A=A$, any net $\left\{2 x_{\alpha}\right\} \rightarrow \theta$ implies $\left\{x_{\alpha}\right\} \rightarrow \theta$, and $U_{J}=(\theta)$ for a closed Jordan ideal, $U$, of $S$ if, and only if, $U=S$. Let $I$ be a self-adjoint ideal of $A$ with $\mathscr{L}(I)=(\theta)$. Then $A^{3} \leqq \bar{I}$.

Let $V$ be the additive subgroup of $S$ generated by the elements $x+x^{*}$ where $x \in I$. As $\left(x+x^{*}, s\right)_{J}=(x, s)_{J}+\left(x^{*}, s\right)_{J}$ we have $V$ as a Jordan ideal of $S$. Clearly, $I \cong B_{V}=\left\{b \mid b a+a^{*} b^{*} \in V\right\}$. Thus, $\mathscr{L}(V)=\mathscr{L}\left(B_{V}\right) \subseteq \mathscr{L}(I)=(\theta)$. Hence, $S=\bar{V} \cong \overline{I \cap S} \cong \bar{I}$. This means that $a+a^{*}$ and $a a^{*}$ are in $\bar{I}$ for all $a \in A$. Therefore, $a\left(a+a^{*}\right) \in \bar{I}$, concluding that $a^{2} \in \bar{I}$ for all $a \in A$. Therefore, $A^{3} \cong \bar{I}$.

As a corollary we have

COROLlary 13. Let A a topological ring with property $(Y)$. Let $I$ be a closed, self-adjoint two-sided ideal of $A$ with $\mathscr{L}(I)=(\theta)$. Then, $I=A$.

THEOREM 14. Let $U$ be a closed Jordan ideal of $S$ in a topological ring with property $(Y)$ then $B \oplus \mathscr{L}(B)=H$ is two-sided and hence, dense in $A$.

$H$ is a right ideal. We wish to show that it is a left ideal and also self-adjoint. Then, Theorem 12 will apply.

Let $W=(\mathscr{L}(B) \cap S) \oplus B \cap S$. Now, $x \in W_{J}$ implies $x \in(B \cap S)_{J}$. Thus by Corollary 10 and Theorem 8, $x \in \mathscr{L}(B) \cap S$ and so $x^{2}=\theta$. Therefore, $W_{J}$ is a nil Jordan ideal and by Theorem $1, \mathscr{L}(W)=(\theta)$. Thus, $\bar{W}=S$. As $W \subseteq H \cap S$, we conclude that $\bar{H} \cap S=S$. Therefore, $a^{2}=\left(a+a^{*}\right) a-a^{*} a \in \bar{H}$ for all $a \in A$. Linearizing this we have for all $a$ and $c$ in $A$

$$
a c+c a \in \bar{H} .
$$

Hence, if $a \in A, b \in B$ then there exists a net $b_{\alpha}+d_{\alpha} \rightarrow a b+b a$ with 
$b_{\alpha} \in B, d_{\alpha} \in \mathscr{L}(B)$. Thus,

$$
\left(b_{\alpha}-b a\right)+d_{\alpha} \rightarrow a b
$$

and so $a b \in \bar{H}$. As $\mathscr{L}(B)$ is two-sided, we conclude that $\bar{H}$ is a left ideal. However, we have just observed that $a^{2} \in \bar{H}$ for all $a \in A$. By the hypothesis on $A$ we can conclude, as before, that $A=\bar{H}$.

The density of $H$ in Theorem 14 leads to the same sequence of theorems as Theorems 3, 4, and 5 of [1]. We summarize these results as

Theorem 15. Let $U$ be a closed Jordan ideal of $S$ in a topological ring $A$ with property ( $Y$ ) then

(i) $B$ is a self-adjoint two-sided ideal of $A$ with $U \subseteq B$.

(ii) $C=U+\overline{\left[\left\{(u, a)_{L} \mid u \in U, a \in A\right\}\right]}$ is a closed two-sided ideal of $A$ and $C \cap S=U$.

Corollary 16. $A=S \oplus \overline{S, S]+S \circ K}$.

Let $U=S$ in Theorem 15, then $C$ has the form of the right side. By Corollary 6, $\mathscr{L}(S)=(\theta)$ and hence $\mathscr{L}(S+[S, S]+S \circ K)=$ $(\theta)$. Corollary 13 then implies the conclusion.

Corollary 17. $A=S \oplus \overline{S \circ K}$.

We see that $(S \circ S)_{J}=(\theta)$ and from this that $S \circ S$ is dense in $S$. A generator of $[S, S]$ has the form $s t-t s$ where $s$ and $t$ are in $S$. As $t \in \overline{S \circ S}$ we can find a net $y_{\alpha}=\sum_{i=1}^{n_{\alpha}}\left(u_{\alpha_{i}}, v_{\alpha_{i}}\right)_{J} \rightarrow t$ with

$$
u_{\alpha_{i}}, v_{\alpha_{i}} \in S \text {. }
$$

Now,

$$
s y_{\alpha}-y_{\alpha} s \rightarrow s t-t s
$$

Consider a summand,

$$
\left[s,\left(u_{\alpha}, v_{\alpha}\right)_{J}\right]=\left[s, u_{\alpha}\right] \circ v_{\alpha}+u_{\alpha} \circ\left[s, v_{\alpha}\right]
$$

and note that the right side is in $S \circ K$. Therefore, $[S, S] \subseteq \overline{S \circ K}$.

We follow the outline of [4] in showing a decomposition for topological rings with property $(Y)$ which have dense socles (such rings have $A^{3}$ dense in $A$ ). Let $e A$ be a minimal right ideal, then $e^{*} A$ also have this property. Let $\hat{I}$ be the intersection of all closed self-adjoint two-sided ideals containing $e A$. Now, $e \in \hat{I}$ and so $e^{*} \in \hat{I}$, therefore, $A\left(e+e^{*}\right) A \subseteq \hat{I}$, and so equality holds. Let $L$ be a non- 
zero closed self-adjoint ideal of $A$ contained in $\hat{I}$ then $\hat{I}=L$. This follows since either $e A \cap L=(\theta)$ or $e A \cap L=e A$. The latter yields immediately $L=\hat{I}$. While $e A \cap L=(\theta)$ implies that $e$ and hence $e^{*} \in \mathscr{L}(L)$. Thus, $\hat{I} \subseteq \mathscr{L}(L)$ and this leads to a contradiction. Now since $e$ and $e^{*}$ belong to the socle, $\zeta$, then $\hat{I}=\overline{A\left(e+e^{*}\right) A \subseteq \zeta \cap \hat{I}}$. Now $A$ is a semiprime ring so the socle of $\hat{I}, \zeta_{I}$, is $\zeta \cap \hat{I}$. Therefore, $\hat{I}$ has dense socle.

This argument forms the basis of the following theorem.

THEOREM 18. If $A$ is a topological ring with property ( $Y$ ) and dense socle, then $A$ is a topological direct sum of two-sided ideals $\hat{I}$ each of which is an involutionally simple and closed ring with dense socle. Moreover, the symmetric elements in $\hat{I}$ form a simple closed Jordan ideal.

We have shown that each $\hat{I}$ is a closed self-adjoint ideal and involutionally minimal closed in $A$. Now, in a semiprime ring it is known that every nonzero ideal of $\hat{I}$ contains a nonzero ideal of $A$, and therefore, $\hat{I}$ is involutionally closed simple as a ring. The density of the socle together with the minimal property of $\hat{I}$ guarantees the direct topological sum. We need only see that $U=\left\{s \in I \mid s^{*}=s\right\}$, the set of symmetric elements of $\hat{I}$, is simple Jordan. Let $G \neq(\theta)$ be a closed Jordan ideal of $U$. As $U \oplus U_{J}$ is dense in $S$ then $G \circ S=$ $G \circ\left(\overline{U+U_{J}}\right) \subseteq \overline{G \circ U} \sqsubseteq G$. That is, $G$ is a closed Jordan ideal of $A$. Letting $E$ be the closure of the subgroup generated by

$$
\left\{(a, g)_{L} \mid a \in A, g \in G\right\},
$$

we conclude from Theorem 15 that $G+E$ is an involutionally closed, nonzero ideal of $\hat{I}$. Thus, $G=U$ completing the argument.

Finally, in this section we exhibit a topological ring with property $(Y)$ which is not an annihilator ring. Consider the ring of polynomials in noncommuting indeterminates over $C$, the complex numbers, modulo the ideal generated by $x y-y x+1$. This ring is known to be a simple ring, of characteristic 0 , with identity and involution which, furthermore, is an integral domain (not a division ring). The ring with discrete topology becomes a topological ring with property $(Y)$ as $S$, because of simplicity, is a simple Jordan ideal. It is not an annihilator ring since it is an integral domain.

It should be noted that Theorem 15 holds for the annihilator rings of [1] as well.

$S$-Dual rings. Following in the vein of dual rings, as defined in 
[3], we define a ring $A$ to be an $S$-dual ring if, and only if, $A$ is a semiprime topological ring with the following property:

(i) $A=2 A$, any net $\left\{2 x_{\alpha}\right\} \rightarrow \theta$ implies $\left\{x_{\alpha}\right\} \rightarrow \theta$;

(ii) $A$ has continuous involution;

(iii) $\left(U_{J}\right)_{J}=U$ for a closed Jordan ideal $U$ of $S$.

We note quickly that $S_{J}=(\theta)$ by Corollary 6 and Theorem 8 . Thus, $\left(S_{J}\right)_{J}=S$ and so if $A^{3}$ is dense in $A$ then we see $S$-dual implies property $(Y)$.

THEorem 19. Let $A$ be an S-dual ring and $U$ be a closed Jordan ideal of $S$. Then, $B$ is a two-sided self-adjoint ideal and $A^{3}$ is contained in $\overline{B+\mathscr{L}(B)}$.

By Corollary 10, $U=B \cap S$. One readily observes that $V=$ $\left\{b+b^{*} \mid b \in B\right\}$ is a Jordan ideal of $S$. Let $x \in U_{J}$. Then, by Theorems 5 and $8, x \in \mathscr{L}(B)$. For $b \in B, x b^{*}=(b x)^{*} \in(B \cap \mathscr{L}(B))^{*}=(\theta)$. Thus, $x \in V_{J}$ or $U_{J} \subseteq V_{J}$. As, $U \subseteq V$, we conclude that $\bar{V}=U$. Thus, for all $b \in B, b+b^{*} \in U \subseteq B$, and so, $b^{*} \in B$. Thus, $B$ is selfadjoint and hence a two-sided ideal. $H=B \oplus \mathscr{L}(B)$ then has the property that $\mathscr{L}(H)=(\theta)$. Applying Theorem 12 we have the desired conclusion.

Corollary 20. Let $A$ be an S-dual ring. Then every closed Jordan ideal, $U$, of $S$ is the intersection of $S$ with a closed two-sided ideal, $I$, of $A$.

The proof is immediate upon setting $I=B$ in Theorem 19 .

Idempotents related to Jordan ideals.

THEOREM 21. Let $A$ be a semiprime ring, let $U$ be a Jordan ideal of $S$. Then either $U \subset \zeta^{a}$ (the annihilator of the socle) or $U$ contains an idempotent.

Let $U \neq(\theta)$. If $e$ is a minimal idempotent then $e B=e A$ or $e B=$ $(\theta)$. If the latter is true for all $e$ then $B \subseteq \zeta^{a}$. Also, $\zeta \subseteq \mathscr{L}(B)=$ $\mathscr{L}(U)$ and therefore $U \subseteq \zeta^{a}$. Assume that $B \nsubseteq \zeta^{2}$ then it is well known that $B$ contains a minimal idempotent $e$. Now, if $e$ is symmetric then $(e,(1 / 2) e)_{J}=e \in U$ and the proof is complete. Thus, assume $e$ is not in $S$, then $e^{*} B=e^{*} A$ (else $e^{*} \in \mathscr{L}(B)$ and as the latter is self-adjoint, $e \in \mathscr{L}(B) \cap B=(\theta)$ ) as this ideal is minimal. Also, $\left(e+e^{*}\right) e \in B$ and so $e^{*} e \in B$. Thus, either $e^{*} e A=e^{*} A$ or $e^{*} e=\theta$. 
Case 1. $e^{*} e A=e^{*} A$. This implies that $e^{*} e \neq \theta$. Let

$$
T=\left\{t \in e^{*} A \mid e^{*} e t=\theta\right\} \text {. Then, } I=e^{*} A \text { or } T=(\theta) .
$$

Subcase 1. If $T=e^{*} A$, then $e^{*} e e^{* *}=\theta$. Now suppose $e e^{*} A \neq(\theta)$, then $e e^{*} A=e A$ and so $(\theta)=e^{*} e A=e^{*} A$, a contradiction. Therefore, $e e^{*}=\theta$. Summarizing we have $e$ and $e^{*} e \in B, e e^{*}=\theta$. Thus,

$$
\left(e+e^{*} e, e\right)_{J}=e+e^{*}+2 e^{*} e \in U
$$

and the square

$$
\left(e+e^{*}+2 e^{*} e\right)^{2}=e+3 e^{*} e+e^{*}+2 e^{*} e \in U .
$$

Thus, subtracting (III) from twice (II) we have that the symmetric idempotent $e+e^{*}-e^{*} e \in U$.

Subcase 2. $T=(\theta)$ or $e^{*} e e^{*} A=e^{*} A$. Thus, there exists $z \in e^{*} A$ such that $e^{*} e z=e^{*} e$ and so that for all $y \in e^{*} A, z y=y$. In particular, $\mathrm{ze}^{*}=e^{*}$ or, applying the involution, $e=e z^{*}$. It now follows that $e^{*} e\left(z-z z^{*}\right)=\theta$. Thus, $z-z z^{*} \in T$, and hence $z$ is a symmetric idempotent. As $e^{*} A=e^{*} e A$ and $e^{*} e \in B$ we have $z \in B$. But, then $z=$ $(z,(1 / 2) z)_{J} \in U$. The desired conclusion.

Case 2. $e^{*} e A=(\theta)$ which means that $e^{*} e=\theta$. Now, as noted $e^{*} \notin \mathscr{L}(B)$ so $e^{*} B=e^{*} A$. Hence, there exists a $b \in B$ such that $e^{*}=$ $e^{*} b$. Now, $\left(e+e^{*}\right) b \in B$ and as $e b \in B$ we conclude $e^{*} \in B$. We are now in the same position to argue on $e^{*}$ as we did previously on $e$, and we conclude that the theorem is proved unless it is also the case that $e e^{*}=\theta$. We immediately see that under these conditions $e+e^{*} \in B$ and is symmetric idempotent. To complete the argument we note that

$$
e+e^{*}=\left(e+e^{*}, \frac{1}{2}\left(e+e^{*}\right)\right)_{J} \in U
$$

CoROllaRy 22. Let $A$ be a semiprime ring, let $\zeta^{a}=(\theta)$, then every nonzero Jordan ideal of $S$ contains an idempotent.

The author wishes to thank the referee for his suggestions relative to the shortening of several arguments in this paper.

\section{REFERENCES}

1. W. E. Baxter, On rings with proper involution, Pacific J. Math. 27 (1968), 1-12.

2. I. N. Herstein, Certain aspects of rings with involution, University of Chicago Lecture Notes, 1967. 
3. I. Kaplansky, Dual rings, Ann. of Math. 49 (1948), 689-701.

4. B. Yood, Ideals in topological rings, Canad. J. Math. 16 (1964), 28-45.

Received November 1, 1968.

UNIVERSITY OF DELAWARE 



\section{PACIFIC JOURNAL OF MATHEMATICS}

\section{EDITORS}

H. ROYDEN

Stanford University

Stanford, California

\section{RichaRd PIERCE}

University of Washington Seattle, Washington 98105
J. DugundJI

Department of Mathematics

University of Southern California

Los Angeles, California 90007

BASIL GORDON

University of California

Los Angeles, California 90024

\section{ASSOCIATE EDITORS}
E. F. BECKENBACH
B. H. NeUMANN
F. WOLF
K. YOSHIDA

\section{SUPPORTING INSTITUTIONS}

UNIVERSITY OF BRITISH COLUMBIA CALIFORNIA INSTITUTE OF TECHNOLOGY

UNIVERSITY OF CALIFORNIA

MONTANA STATE UNIVERSITY

UNIVERSITY OF NEVADA

NEW MEXICO STATE UNIVERSITY

OREGON STATE UNIVERSITY

UNIVERSITY OF OREGON

OSAKA UNIVERSITY

UNIVERSITY OF SOUTHERN CALIFORNIA

\author{
STANFORD UNIVERSITY \\ UNIVERSITY OF TOKYO \\ UNIVERSITY OF UTAH \\ WASHINGTON STATE UNIVERSITY \\ UNIVERSITY OF WASHINGTON \\ AMERICAN MATHEMATICAL SOCIETY \\ CHEVRON RESEARCH CORPORATION \\ TRW SYSTEMS \\ NAVAL WEAPONS CENTER
}

The Supporting Institutions listed above contribute to the cost of publication of this Journal, but they are not owners or publishers and have no responsibility for its content or policies.

Mathematical papers intended for publication in the Pacific Journal of Mathematics should be in typed form or offset-reproduced, double spaced with large margins. Underline Greek letters in red, German in green, and script in blue. The first paragraph or two must be capable of being used separately as a synopsis of the entire paper. It should not contain references to the bibliography. Manuscripts, in duplicate if possible, may be sent to any one of the four editors. Please classify according to the scheme of Math. Rev. 36, 1539-1546. All other communications to the editors should be addressed to the managing editor, Richard Arens, University of California, Los Angeles, California, 90024.

50 reprints are provided free for each article; additional copies may be obtained at cost in multiples of 50 .

The Pacific Journal of Mathematics is published monthly. Effective with Volume 16 the price per volume (3 numbers) is $\$ 8.00$; single issues, $\$ 3.00$. Special price for current issues to individual faculty members of supporting institutions and to individual members of the American Mathematical Society: $\$ 4.00$ per volume; single issues $\$ 1.50$. Back numbers are available.

Subscriptions, orders for back numbers, and changes of address should be sent to Pacific Journal of Mathematics, 103 Highland Boulevard, Berkeley, California, 94708.

PUBLISHED BY PACIFIC JOURNAL OF MATHEMATICS, A NON-PROFIT CORPORATION

Printed at Kokusai Bunken Insatsusha (International Academic Printing Co., Ltd.), 7-17. Fujimi 2-chome, Chiyoda-ku, Tokyo, Japan. 


\section{Pacific Journal of Mathematics}

\section{Vol. 30, No. $3 \quad$ November, 1969}

Willard Ellis Baxter, Topological rings with property $(Y) \ldots \ldots \ldots \ldots . \ldots 5$

Sterling K. Berberian, Note on some spectral inequalities of $C . R$.

Putnam ..................................... 573

David Theodore Brown, Galois theory for Banach algebras . ........... 577

Dennis K. Burke and R. A. Stoltenberg, A note on p-spaces and Moore spaces ........................................ 601

Rafael Van Severen Chacon and Stephen Allan McGrath, Estimates of positive contractions....................................... 609

Rene Felix Dennemeyer, Conjugate surfaces for multiple integral problems in the calculus of variations ........................... 621

Edwin O. Elliott, Measures on countable product spaces.............. 639

John Moss Grover, Covering groups of groups of Lie type .............. 645

Charles Lemuel Hagopian, Concerning semi-local-connectedness and cutting in nonlocally connected continua .................. 657

Velmer B. Headley, A monotonicity principle for eigenvalues ........... 663

John Joseph Hutchinson, Intrinsic extensions of rings . . . . . . . . . . . . . 669

Harold H. Johnson, Determination of hyperbolicity by partial

prolongations .................................. 679

Tilla Weinstein, Holomorphic quadratic differentials on surfaces in $E^{3} \ldots 697$

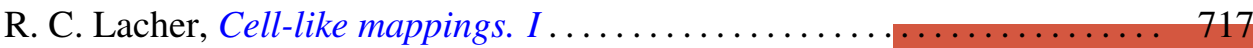

Roger McCann, A classification of centers

Curtis L. Outlaw, Mean value iteration of nonexpansive mappings in a

Banach space...

Allan C. Peterson, Distribution of zeros of solutions of a fourth order

differential equation.

Bhalchandra B. Phadke, Polyhedron inequality and strict convexity .. 765 Jack Wyndall Rogers Jr., On universal tree-like continua .

Edgar Andrews Rutter, Two characterizations of quasi-Frobenius rings

G. Sankaranarayanan and C. Suyambulingom, Some renewal theorems concerning a sequence of correlated random variables...

Joel E. Schneider, A note on the theory of primes........ . .

Richard Peter Stanley, Zero square rings .................

Edward D. Tymchatyn, The 2-cell as a partially ordered space

Craig A. Wood, On general Z.P.I.-rings................ 